\title{
Brane World black holes in Teleparallel Theory Equivalent to General Relativity and their Killing vectors, Energy, Momentum and Angular-Momentum*
}

\author{
Gamal G.L. Nashed \\ Centre for Theoretical Physics, The British University in Egypt, El-Sherouk City, \\ Misr - Ismalia Desert Road, Postal No. 11837, P.O. Box 43, Egypt. \\ e-mail:gamal.nasshed@bue.edu.eg
}

The energy-momentum tensor, which is coordinate independent, is used to calculate en'ergy, momentum and angular-momentum of two different tetrad fields. Although, the two 'tetrad fields reproduce the same space-time their energies are different. Therefore, a regularized expression of the gravitational energy-momentum tensor of the teleparallel equivalent of general relativity, (TEGR), is used to make the energies of the two tetrad fields equal. The definition of the gravitational energy-momentum is used to investigate the energy within the external event horizon. The components of angular-momentum associated with these space-times are calculated. In spite that we use a static space-times, we get a non-zero component of angular-momentum! Therefore, we derive the killing vectors associated with these space-times using the definition of the Lie derivative of a second rank tensor in the framework of the TEGR to make the picture more clear.

Keywords: Teleparallel equivalent of general relativity, Brane world black holes, Gravitational energy-momentum tensor, Regularized expression of the gravitational energy-momentum.

PACS numbers: 0440, 0450, 0455, 0490.

*Mathematics Department, Faculty of Science, Ain Shams University, Cairo, Egypt. 


\section{Introduction}

Quantum mechanics and general relativity are two very successful and well validated theories within their own domains. The problem is that there is no way to unify them into a single consistent theory. One of the most promising models of unification is string theory. Two classes of strings are the closed and open strings. Gravity is described by closed strings and matter is described by open strings. In non-pertubative string theory there exist extended objects known as D-branes. These are surfaces where the open strings must start and finish. This provides an alternative to Kaluza-Klein [1] approach, where matter penetrates the extra dimensions, leading to strong constraints from collider physics.

A model that captures some of the essential features of the dimensional reduction of 11-dimensional supergravity proposed by Hor ava and Witten [2] is introduced [3]. The gravitational field on the brane is defined by the modified Einstein equations given by Shiromizu, Maeda and Sasaki [4] from 5-dimensional gravity using the Gauss and Codazzi equations [5].

$$
G_{\mu \nu}=-\Lambda_{4} \delta_{\mu \nu}-K_{4}^{2} T_{\mu \nu}-K_{5}^{4} \Pi_{\mu \nu}-E_{\mu \nu}, \quad \Lambda_{4}=\frac{\kappa_{5}^{2}}{2}\left(\Lambda_{5}+\frac{\varrho^{2} \kappa_{5}^{2}}{6}\right)
$$

where $\Lambda_{4}$ is the 4-dimensional cosmological constant expressed in terms of the 5-dimensional cosmological constant $\Lambda_{5}, G_{\mu \nu}$ is the Einstein 4-dimensional tensor, $\varrho$ is the brane tension, $\kappa_{4}^{2}=8 \pi G_{N}=\frac{\varrho \kappa_{5}^{2}}{6 \pi}$ is the 4-dimensional gravitational constant, $G_{N}$ is the Newton's constant of gravity, $T_{\mu \nu}$ is the stress energy tensor of matter confined on the brane, $\Pi_{\mu \nu}$ is a tensor quadratic in $T_{\mu \nu}$ obtained from the 5 -dimensional metric across the brane

$$
2 \Pi_{\mu}{ }^{\nu}=T_{\mu}{ }^{\beta} T_{\beta}{ }^{\nu}-T T_{\mu}{ }^{\nu}-\delta_{\mu}{ }^{\nu}\left(T_{\rho \epsilon} T^{\rho \epsilon}-\frac{T^{2}}{2}\right)
$$

where $T$ is the trace of the stress energy tensor of matter and $E_{\mu \nu}$ is the electric part of the 5-dimensional Weyle tensor projected onto the brane. In proper 5-dimensional coordinate $E_{\mu \nu}=\delta_{\mu}{ }^{M} \delta_{\nu}{ }^{N} C_{M N K L} n^{K} n^{L}$ where $M, N \cdots$ are 5-dimensional indices and $n^{M}$ is the unit normal to the brane [6]. Here we are going to select a class of spherically symmetric black holes without specifying $E_{\mu \nu}$.

Among various attempts to overcome the problems of quantization and the existence of singular solution in Einstein's general relativity (GR), gauge theories of gravity are of special attractive, as they based on the concept of gauge symmetry which has been very successful in the foundation of other fundamental interactions. The importance of the poincaré symmetry in particle physics leads one to consider the poincaré gauge theory (PGT) as a natural framework for description of the gravitational phenomena [7] [13]. Basic gravitational variables in PGT are the tetrad field $e^{a}{ }_{\mu}$ and the Lorentz connection $A^{a b}{ }_{\mu}$, which are associated to the translation and Lorentz subgroups of the poincaré group, respectively. These gauge fields are coupled to the energy-momentum and spin of matter fields, and their field strengths are geometrically identified with the torsion and the curvature. The spacetime of the PGT turns out to be Riemann-Cartan space $U_{4}$, equipped with metric and linear, metric compatible connection. 
General geometric arena of PGT, the Riemann-Cartan space $U_{4}$, may be a priori restricted by imposing certain conditions on the curvature and the torsion. Thus, Einstein's GR is defined in Riemann space $V_{4}$, which is obtained from $U_{4}$ by the requirement of vanishing torsion. Another interesting limit of PGT is the teleparallel or Weitzenböck geometry $T_{4}$. The vanishing of the curvature means that parallel transport is path independent. The teleparallel geometry is, in sense, complementary to Riemannian: curvature vanishes, and torsion remains to characterize the parallel transport. Of particular importance for the physical interpretation of the teleparallel geometry the fact that there is a one-parameter family of teleparallel Lagrangians which is empirically equivalent to GR $[12,14,15]$. For the parameter value $B=1 / 2$ the Lagrangian of the theory coincides, modulo a four-divergence, with the Einstein-Hilbert Lagrangian, and defines (TEGR).

The search for a consistent expression for the gravitating energy and angular-momentum of a self-gravitating distribution of matter is undoubtedly a long-standing problem in GR. It is believed that the energy of the gravitational field is not localizable, i.e., defined in a finite region of the space. The gravitational field does not possess the proper definition of an energy momentum tensor and one usually defines some energy-momentum and angularmomentum as Bergmann [16] or Landau-Lifschitz [17] which are pseudo-tensors and depend on the second derivative of the metric tensor. These quantities can be annulled by an adequate transformation of coordinate. They $[16,17]$ justify the results as being consistent with Einstein's principle of equivalence. In this principle, it can be always find a small region of space-time, where it prevails Minkowski space-time. In such a space-time, energy of the gravitational field is null. Therefore, it is only possible to define the energy of the gravitational filed in whole space-time region and not only in a small region. The Einstein's GR can also be reformulated in the context of teleparallel geometry [18] [27]. In this geometrical setting the dynamical field quantities correspond to orthonormal tetrad field $e^{a}{ }_{\mu}{ }^{*}$ (a, $\mu$ are $\mathrm{SO}(3,1)$ and space-time indices, respectively). The teleparallel geometry is a suitable framework to address the notions of energy, momentum and angular-momentum of any space-time that admits a $3+1$ foliation [28]. Therefore, we consider the TEGR.

Hamiltonian formulation of TEGR, in Schwinger's time gauge [29], has been established [30]. This formulation is an alternative teleparallel geometric description to Einstein GR theory. An essential feature of this Hamiltonian formulation is that one can defines energy of the gravitational field by means of an adequate interpretation of the Hamiltonian constraint. Several configurations of the gravitational energy were investigated with success [30, 31]. TEGR can be understood as a gauge theory for the translation group [27]. In this approach, the gravitational interaction is described by a force similar to the Lorentz force equation of electrodynamics, with torsion playing the role of force. Regge and Teitelboim [32] obtained a Hamiltonian formalism for GR that is manifestly invariant under Ponicaré transformations at infinity by introducing ten new pairs of canonical variables, which yield ten surface integrals to the total Hamiltonian. The subsequent analysis given by York [33] showed that a proper definition for the gravitational angular-momentum requires a suitable asymptotic behavior of the spatial components of the Ricci tensor. A careful analysis of the exact form of the boundary conditions needed to define the energy, momentum and angular-momentum of the gravitational field has been carried out by Beig and ó Murchadha [34] and by Szabados [35] who found the necessary conditions that yield a finite value for the above quantities. In these

${ }^{*}$ space-time indices $\mu, \nu, \cdots$ and $\mathrm{SO}(3,1)$ indices $\mathrm{a}, \mathrm{b} \cdots$ run from 0 to 3 . Time and space indices are indicated to $\mu=0, i$, and $a=(0),(i)$. 
analysis the poincaré algebra are realized at the spacelike infinity. These are transformations of the Cartesian coordinates in the asymptotic region of the space-time.

The Hamiltonian formulation of an arbitrary teleparallel theories using Schwinger's time gauge is established [36, 37]. In this formulation it is shown that the TEGR is the only viable consistent teleparallel theory of gravity. Maluf and Rocha [38] established a theory in which Schwinger's time gauge has not been incorporated in the geometry of absolute parallelism. In this formulation, the definition of the gravitational angular-momentum arises by suitably interpreting the integral form of the constraint equation $\Gamma^{a b}=0$. This definition has been successfully applied to the gravitational field of a thin, slowly rotating mass shell [39] and for the three-dimensional BTZ black hole [40]. Shanxian et al. [41] have calculated the energy of general 4-Dim. stationary axisymmetric spacetime in the teleparallel geometry.

Definitions for the gravitational energy in the context of the TEGR have already been proposed in the literature. An expression for the gravitational energy arises from the surface term of the total Hamiltonian is given in Ref. [42]. In Ref. [43], a similar quantity is suggested. Both expressions are equivalent to the integral form of the total divergences of the Hamiltonian density developed in Ref. [38]. The three expressions yield the same value for the total energy of the gravitational field. However, since these three expressions contain the lapse function in the integrand, non of them is suitable to the calculation of the irreducible mass of the Kerr black hole because the lapse function vanishes on the external event horizon of the black hole [30]. The energy expressions of References [42], [43] are not to be applied to a finite surface integration; rather they yield the total energy of the space-time [30].

The Localization of gravitational energy-momentum remains an important problem in GR. Using the standard methods many famous researchers each found their own expression. None of these expressions is covariant, they are all reference frame dependent (referred to as pseudotensors). This feature can be understood in terms of the equivalence principle: gravity cannot be detected at a point, so it cannot have a point-wise defined energy-momentum density. Now there is another way to address this difficulty. The new idea is the quasi-local energy-momentum is associated with a closed 2 surface surrounding a region. A good quasilocal approach is in terms of the Hamiltonian [25]. Then the Hamiltonian boundary term determines the quasi-local quantities. In fact this approach includes all the traditional pseudotensors $[25,44]$. They are each generated by a superpotential which can serve as special type of Hamiltonian boundary term. A good energy-momentum expression for gravitating systems should satisfy a variety of requirements, including giving the standard values for the total quantities for asymptotically flat space and reducing to the material energy-momentum in proper limit. No entirely expression has yet been identified. One of the most restrictive requirements is positivity. A general positivity proof is very difficult [45]. For more details of the topic of quasi-local approach a very nice review article is given in [46].

The definition of $P^{a}$ is invariant under global $S O(3,1)$ transformations. It has been argued elsewhere [47] that it makes sense to have a dependence of $P^{a}$ on the frame. The energy-momentum in classical theories of particles and fields does not depend on the frame, and it has been asserted that such dependence is a natural property of the gravitational energy-momentum. The total energy of a relativistic body, depends on the frame. It is assumed that a set of tetrads fields is adapted to an observer in the space-time determined by the metric tensor $g_{\mu \nu}$. 
We investigate the irreducible mass $M_{i r r}$ of the brane world black holes. It is the total mass of the black hole at the final stage of Penrose's process of energy extraction, considering that the maximum possible energy is extracted. It is also related to the energy contained within the external event horizon $E\left(r_{+}\right)$of the black hole (the surface of the constant radius $r=r_{+}$defines the external event horizon). Every expression for local or quasi-local gravitational energy must necessary yield the value of $E\left(r_{+}\right)$in close agreement with $2 M_{i r r}$, since we know beforehand the value of the latter as a function of the initial angular-momentum of the black hole [48]. The evolution of $2 M_{i r r}$ is a crucial test for any expression for the gravitational energy. $E\left(r_{+}\right)$has been obtained by means of different energy expressions in Ref. [49]. The gravitational energy used in this article is the only one that yields a satisfactory value for $E\left(r_{+}\right)$and that arises in the framework of the Hamiltonian formulation of the gravitational field.

It is the aim of the present paper to find asymptotically flat solutions with spherical symmetry in the TEGR for the gravitational such that $\Lambda_{4}=T_{\mu \nu}=0$. In this case we can treat Eq. (1) as conventional Einstein equations with an effective stress energy tensor $\hat{T}_{\mu \nu}$. Using the energy-momentum tensor [30], we calculate the energy, momentum, angularmomentum associated with these solutions. We also derive the killing vectors related to these solutions to discuss the different results of energy, momentum, angular-momentum.

The paper is organizing as follows. In $\S 2$, we briefly review the TEGR theory, the energy-momentum tensor and the angular-momentum. The two different tetrad fields with two unknown functions are studied in $\S 2$. In $\S 3$, we use the regularized expression for the gravitational energy-momentum to recalculate the energy. In $\S 4$, we derive explicitly the killing vectors related to these two tetrad fields, using the definition of the Lie derivative of a second rank tensor in the framework of the TEGR, to discuss the different results we obtained for the energy and angular-momentum. Final section is devoted to discussion and conclusion.

\section{The TEGR for gravitation, energy, momentum, angular-momentum}

In a space-time with absolute parallelism the parallel vector fields $e_{a}{ }^{\mu}$ define the nonsymmetric affine connection

$$
\Gamma_{\mu \nu}^{\lambda} \stackrel{\text { def. }}{=} e_{a}^{\lambda} e_{\mu, \nu}^{a}
$$

where $e_{a \mu, \nu}=\partial_{\nu} e_{a \mu}{ }^{*}$. The curvature tensor defined by $\Gamma_{\mu \nu}^{\lambda}$, given by Eq. (3), is identically vanishing. The metric tensor $g_{\mu \nu}$ is defined by

$$
g_{\mu \nu} \stackrel{\text { def. }}{=} \eta_{a b} e^{a}{ }_{\mu} e^{b}
$$

with $\eta_{a b}=(-1,+1,+1,+1)$ is the metric of Minkowski space-time.

\footnotetext{
*space-time indices $\mu, \nu, \cdots$ and $\mathrm{SO}(3,1)$ indices $\mathrm{a}, \mathrm{b} \cdots$ run from 0 to 3 . Time and space indices are indicated to $\mu=0, i$, and $a=(0),(i)$.
} 
The Lagrangian density for the gravitational field in the TEGR, in the presence of matter fields, is given $\mathrm{by}^{\dagger}[30]$

$$
\mathcal{L}_{G}=e L_{G}=-\frac{e}{16 \pi}\left(\frac{T^{a b c} T_{a b c}}{4}+\frac{T^{a b c} T_{b a c}}{2}-T^{a} T_{a}\right)-L_{m}=-\frac{e}{16 \pi} \Sigma^{a b c} T_{a b c}-L_{m}
$$

where $e=\operatorname{det}\left(e^{a}{ }_{\mu}\right)$. The tensor $\Sigma^{a b c}$ is defined by

$$
\Sigma^{a b c} \stackrel{\text { def. }}{=} \frac{1}{4}\left(T^{a b c}+T^{b a c}-T^{c a b}\right)+\frac{1}{2}\left(\eta^{a c} T^{b}-\eta^{a b} T^{c}\right)
$$

$T^{a b c}$ and $T^{a}$ are the torsion tensor and the basic vector field defined by

$$
T_{\mu \nu}^{a} \stackrel{\text { def. }}{=} e_{\lambda}^{a} T_{\mu \nu}^{\lambda}=\partial_{\mu} e_{\nu}^{a}-\partial_{\nu} e_{\mu}^{a}, \quad T_{b c}^{a} \stackrel{\text { def. }}{=} e_{b}{ }^{\mu} e_{c}{ }^{\nu} T_{\mu \nu}^{a} \quad \quad T^{a} \stackrel{\text { def. }}{=} T_{b}^{b}{ }_{b}^{a} .
$$

The quadratic combination $\Sigma^{a b c} T^{a b c}$ is proportional to the scalar curvature $R(e)$, except for a total divergence term [30]. $L_{m}$ represents the Lagrangian density for matter fields.

The gravitational field equations for the system described by $L_{G}$ are the following

$$
e_{a \lambda} e_{b \mu} \partial_{\nu}\left(e \Sigma^{b \lambda \nu}\right)-e\left(\Sigma_{a}^{b \nu} T_{b \nu \mu}-\frac{1}{4} e_{a \mu} T_{b c d} \Sigma^{b c d}\right)=\frac{1}{2} \kappa e T_{a \mu}
$$

where

$$
\frac{\delta L_{m}}{\delta e^{a \mu}} \equiv e T_{a \mu}
$$

It is possible to prove by explicit calculations that the left hand side of the symmetric field equations (8) is exactly given by [30]

$$
\frac{e}{2}\left[R_{a \mu}(e)-\frac{1}{2} e_{a \mu} R(e)\right]
$$

The axial-vector part of the torsion tensor $a_{\mu}$ is defined by

$$
a_{\mu} \stackrel{\text { def. }}{=} \frac{1}{6} \epsilon_{\mu \nu \rho \sigma} T^{\nu \rho \sigma}=\frac{1}{3} \epsilon_{\mu \nu \rho \sigma} \gamma^{\nu \rho \sigma}, \quad \text { where } \quad \epsilon_{\mu \nu \rho \sigma} \stackrel{\text { def. }}{=} \sqrt{-g} \delta_{\mu \nu \rho \sigma},
$$

and $\delta_{\mu \nu \rho \sigma}$ being completely antisymmetric and normalized as $\delta_{0123}=-1$.

In the context of Einstein's GR, rotational phenomena is certainly not a completely understood issue. The prominent manifestation of a purely relativistic rotation effect is the dragging of inertial frames. If the angular-momentum of the gravitational field of isolated system has a meaningful notion, then it is reasonable to expect the latter to be somehow related to the rotational motion of the physical sources.

The angular-momentum of the gravitational field has been addressed in the literature by means of different approaches. The oldest approach is based on pseudotensors [16, 17],

\footnotetext{
${ }^{\dagger}$ Throughout this paper we use the relativistic units , $c=G=1$ and $\kappa=8 \pi$.
} 
out of which angular-momentum superpotentials are constructed. An alternative approach assumes the existence of certain Killing vector fields that allow the construction of conserved integral quantities [50]. Finally, the gravitational angular-momentum can also be considered in the context of Poincaré gauge theories of gravity [51], either in the Lagrangian or in the Hamiltonian formulation. In the latter case it is required that the generators of spatial rotations at infinity have a well defined functional derivatives. From this requirement a certain surface integral arises, whose value is interpreted as the gravitational angular-momentum.

The Hamiltonian formulation of TEGR is obtained by establishing the phase space variables. The Lagrangian density does not contain the time derivative of the tetrad component $e_{a 0}$. Therefore, this quantity will arise as a Lagrange multiplier [52]. The momentum canonically conjugated to $e_{a i}$ is given by $\Pi^{a i}=\delta L / \delta \dot{e}_{a i}$. The Hamiltonian formulation is obtained by rewriting the Lagrangian density in the form $L=p \dot{q}-H$, in terms of $e_{a i}$, $\Pi^{a i}$ and the Lagrange multipliers. The Legendre transformation can be successfully carried out and the final form of the Hamiltonian density has the form [28]

$$
H=e_{a 0} C^{a}+\alpha_{i k} \Gamma^{i k}+\beta_{k} \Gamma^{k}
$$

plus a surface term. Here $\alpha_{i k}$ and $\beta_{k}$ are Lagrange multipliers that are identified as

$$
\alpha_{i k}=\frac{1}{2}\left(T_{i 0 k}+T_{k 0 i}\right) \quad \text { and } \quad \beta_{k}=T_{00 k}
$$

and $C^{a}, \Gamma^{i k}$ and $\Gamma^{k}$ are first class constraints. The Poisson brackets between any two field quantities $F$ and $G$ is given by

$$
\{F, G\}=\int d^{3} x\left(\frac{\delta F}{\delta e_{a i}(x)} \frac{\delta G}{\delta \Pi^{a i}(x)}-\frac{\delta F}{\delta \Pi^{a i}(x)} \frac{\delta G}{\delta e_{a i}(x)}\right)
$$

We recall that the Poisson brackets $\left\{\Gamma^{i j}(x), \Gamma^{k l}(x)\right\}$ reproduce the angular-momentum algebra $[30]$.

The constraint $C^{a}$ is written as $C^{a}=-\partial_{i} \Pi^{a i}+h^{a}$, where $h^{a}$ is an intricate expression of the field variables. The integral form of the constraint equation $C^{a}=0$ motivates the definition of the gravitational energy-momentum $P^{a}$ four-vector [30]

$$
P^{a}=-\int_{V} d^{3} x \partial_{i} \Pi^{a i}
$$

where $V$ is an arbitrary volume of the three-dimensional space. In the configuration space we have

$$
\begin{gathered}
\Pi^{a i}=-4 \kappa \sqrt{-g} \Sigma^{a 0 i} \quad \text { with } \quad \partial_{\nu}\left(\sqrt{-g} \Sigma^{a \lambda \nu}\right)=\frac{1}{4 \kappa} \sqrt{-g} e_{\mu}^{a}\left(t^{\lambda \mu}+T^{\lambda \mu}\right) \text { where } \\
t^{\lambda \mu}=\kappa\left(4 \Sigma^{b c \lambda} T_{b c}{ }^{\mu}-g^{\lambda \mu} \Sigma^{b c d} T_{b c d}\right) .
\end{gathered}
$$

The emergence of total divergences in the form of scalar or vector densities is possible in the framework of theories constructed out of the torsion tensor. Metric theories of gravity 
do not share this feature. By making $\lambda=0$ in Eq. (14) and identifying $\Pi^{a i}$ in the left side of the latter, the integral form of Eq. (14) is written as

$$
P^{a}=\int_{V} d^{3} x \sqrt{-g} e^{a}{ }_{\mu}\left(t^{0 \mu}+T^{0 \mu}\right)
$$

Eq. (15) suggests that $P^{a}$ is now understood as the gravitational energy-momentum [30]. The spatial component $P^{(i)}$ form a total three-momentum, while temporal component $P^{(0)}$ is the total energy [17].

It is possible to rewrite the Hamiltonian density of Eq. (10) in the equivalent form [39]

$$
H=e_{a 0} C^{a}+\frac{1}{2} \lambda_{a b} \Gamma^{a b}, \quad \text { with } \quad \lambda_{a b}=-\lambda_{b a}
$$

are the Lagrangian multipliers that are identified as $\lambda_{i k}=\alpha_{i k}$ and $\lambda_{0 k}=-\lambda_{k 0}=\beta_{k}$. The constraints $\Gamma^{a b}=-\Gamma^{b a}[28]$ embodies both constraints $\Gamma^{i k}$ and $\Gamma^{k}$ by means of the relation

$$
\Gamma^{i k}=e_{a}^{i} e_{b}^{k} \Gamma^{a b}, \quad \text { and } \quad \Gamma^{k} \equiv \Gamma^{0 k}=e_{a}^{0} e_{b}^{k} \Gamma^{a b}
$$

The constraint $\Gamma^{a b}$ can be reads as

$$
\Gamma^{a b}=M^{a b}+4 \kappa \sqrt{-g} e_{(0)}^{0}\left(\Sigma^{a(0) b}-\Sigma^{b(0) a}\right) .
$$

In similarity to the definition of $P^{a}$, the integral form of the constraint equation $\Gamma^{a b}=0$ motivates the new definition of the space-time angular-momentum. The equation $\Gamma^{a b}=0$ implies

$$
M^{a b}=-4 \kappa \sqrt{-g} e_{c}^{0}\left(\Sigma^{a c b}-\Sigma^{b c a}\right),
$$

Maluf et al. [30, 39] defined

$$
L^{a b}=\int_{V} d^{3} x e_{\mu}^{a} e_{\nu}^{b} M^{\mu \nu}
$$

as the 4-angular-momentum of the gravitational field for an arbitrary volume $\mathrm{V}$ of the threedimensional space. In Einstein-Cartan type theories there also appear constraints that satisfy the Poisson bracket as given by Eq. (12). However, such constraints arise in the form $\Pi^{[i j]}=0$, and so a definition similar to Eq. (20), i.e., interpreting the constraint equation as an equation for the angular-momentum of the field, is not possible. Definition (20) is threedimensional integral. The quantities $P^{a}$ and $L^{a b}$ are separately invariant under general coordinate transformations of the three-dimensional space and under time reparametrizations, which is an expected feature since these definitions arise in the Hamiltonian formulation of the theory. Moreover, these quantities transform covariantly under global $S O(3,1)$ transformations [39].

We will consider two simple configuration of tetrad fields and discuss their physical interpretation as reference frames. The first one in quasi-orthogonal coordinate system can be written as [53]

$$
\begin{aligned}
e_{(0)}{ }^{0} & =A, \quad e_{\alpha}{ }^{0}=C x^{a}, \quad e_{(0)}^{\alpha}=D x^{\alpha} \\
e_{i}{ }^{\alpha} & =\delta_{a}^{\alpha} B+F x^{a} x^{\alpha}+\epsilon_{a \alpha \beta} S x^{\beta},
\end{aligned}
$$


where $A, C, D, B, F$, and $S$ are unknown functions of $r$. It can be shown that the functions $D$ and $F$ can be eliminated by coordinate transformations $[54,55]$, i.e., by making use of freedom to redefine $t$ and $r$, leaving the tetrad field (21) having four unknown functions in the quasi-orthogonal coordinates. Thus the tetrad field (21) without the functions $D$ and $F$ and also without the two functions $C$ and $S$ will be used in the following discussion for the calculations of energy, momentum and angular-momentum but in the spherical coordinate. Therefore, the tetrad field (21) can be written in the spherical coordinates without the functions $D, F, C$ and $S$ as $[55,56]$

$$
\left(e_{1 a}{ }^{\mu}\right)=\left(\begin{array}{cccc}
\frac{1}{A} & 0 & 0 & 0 \\
0 & \frac{B}{\sqrt{r}} \sin \theta \cos \phi & \frac{\cos \theta \cos \phi}{r} & -\frac{\sin \phi}{r \sin \theta} \\
0 & \frac{B}{\sqrt{r}} \sin \theta \sin \phi & \frac{\cos \theta \sin \phi}{r} & \frac{\cos \phi}{r \sin \theta} \\
0 & \frac{B}{\sqrt{r}} \cos \theta & -\frac{\sin \theta}{r} & 0
\end{array}\right) .
$$

The other configuration of tetrad field that has a simple interpretation as a reference frame can has the form

$$
\left(e_{2 a}{ }^{\mu}\right)=\left(\begin{array}{cccc}
\frac{1}{A} & 0 & 0 & 0 \\
0 & \frac{B}{\sqrt{r}} & 0 & 0 \\
0 & 0 & \frac{1}{r} & 0 \\
0 & 0 & 0 & \frac{1}{r \sin \theta}
\end{array}\right) .
$$

The space-time associated with the two tetrad fields (22) and (23) is the same and has the form

$$
d s^{2}=-A^{2} d t^{2}+\frac{r}{B^{2}} d r^{2}+r^{2}\left(d \theta^{2}+\sin ^{2} \theta d \phi^{2}\right)
$$

and the non-vanishing components of the effective stress energy-momentum tensor associated with the space-time given by Eq. (24) are

$$
\begin{gathered}
\hat{T}_{0}{ }^{0}=\hat{\rho}=\frac{1-2 B B^{\prime}}{\kappa_{4}^{2} r^{2}}, \quad \hat{T}_{1}{ }^{1}=\hat{\rho}_{\text {rad }}=\frac{r A-B^{2}\left(A-2 r A^{\prime}\right)}{\kappa_{4}^{2} r^{3} A}, \\
\hat{T}_{2}{ }^{2}=\hat{T}_{3}{ }^{3}=\hat{\rho}_{\text {tang }}=\frac{B\left(A B-2 r A B^{\prime}-r B A^{\prime}-2 r^{2} B A^{\prime \prime}-2 r^{2} A^{\prime} B^{\prime}\right)}{2 \kappa_{4}^{2} r^{3} A} .
\end{gathered}
$$

The two tetrad fields satisfy the field equations (8). Now we are going to calculate the energy, momentum and angular-momentum associated with the two tetrad fields (22) and (23). For asymptotically flat space-times $P^{0}$ yields the ADM energy [57]. In the context of tetrad theories of gravity, asymptotically flat space-times may be characterized by the asymptotic boundary condition

$$
e_{a \mu} \cong \eta_{a \mu}+\frac{1}{2} h_{a \mu}(1 / r)
$$


and by the condition $\partial_{\mu} e^{a}{ }_{\mu}=O\left(1 / r^{2}\right)$ in the asymptotic limit $r \rightarrow \infty$. An important property of tetrad fields that satisfy Eq. (25) is that in the flat space-time limit one has $e^{a}{ }_{\mu}(t, x, y, z)=\delta^{a}{ }_{\mu}$, and therefore the torsion tensor $T_{\mu \nu}^{a}=0$.

We apply Eq. (13) to the tetrad field (22) to calculate the energy content. Calculations are performed in the spherical coordinate. Eqs. (22) and (23) assumed that the reference space is determined by a set of tetrad fields $e^{a}{ }_{\mu}$ for the flat space-time such that the condition $T^{a}{ }_{\mu \nu}=0$ is satisfied. Using Eq. (7) in Eq. (22), the non-vanishing components of the torsion tensor are given by

$$
T^{(0)}{ }_{01}=\frac{A^{\prime}}{A}, \quad T_{12}^{(2)}=\frac{(\sqrt{r}-B)}{r B}=T^{(3)} 13,
$$

and the non-vanishing component of the tensor $T^{(a)}$ is given by

$$
T^{(1)}=\frac{B\left(r B A^{\prime}-2 A\{\sqrt{r}-B\}\right)}{r^{2} A} .
$$

The axial vector associated with Eq. (22) is vanishing identically due to the fact that the tetrad field of Eq. (22) has a spherical symmetry [53].

Now we are going to apply Eq. (6) to the tetrad field (22) using Eqs. (26) and (27) to calculate the energy content. We perform the calculations in the spherical coordinate. The only required component of $\Sigma^{\mu \nu \lambda}$ is

$$
\Sigma^{(0) 01}=-\frac{\sin \theta\{r-\sqrt{r} B\}}{4 \pi} .
$$

Further substituting Eq. (28) in (13) we obtain

$$
P^{(0)}=E=-\oint_{S \rightarrow \infty} d S_{k} \Pi^{(0) k}=-\frac{1}{4 \pi} \oint_{S \rightarrow \infty} d S_{k} e \Sigma^{(0) 0 k}=\{r-\sqrt{r} B\} .
$$

Let us apply expression (13) to the evaluation of the irreducible mass by fixing $V$ to be the volume within the $r=r_{+}$surface where $r_{+}$is the external horizon, i.e., $B=0$. Therefore,

$$
P^{(0)}=E=-\int_{S_{i}} d S_{i} \Pi^{(0) i}=-\int_{S} d \theta d \phi \Pi^{(0) 1}(r, \theta, \phi)
$$

where the surface $S$ is determined by the condition $r=r_{+}$. The expression of $\Pi^{(0) 1}$ will be obtained by considering Eq. (14) using Eq. (6) and Eq. (7). The expression of $\Pi^{(0) 1}(r, \theta, \phi)$ for the tetrad (22) reads

$$
\Pi^{(0) 1}=\frac{\sin \theta\{r-\sqrt{r} B\}}{4 \pi},
$$

integrate Eq. (31) on the surface of constant radius $r=r_{+}$where $r_{+}$is the external horizon of the black hole. On this surface the second term of Eq. (31) vanishes. Therefore, on the surface $r=r_{+}$we get

$$
P^{(0)}=E=r_{+},
$$


a result that is obtained before [30, 46].

Now let us continue to calculate the momentum and angular-momentum associated with the first tetrad field given by Eq. (22). Using Eq. (14) in (22) we get

$$
\Pi^{(1) 1}=0 .
$$

Substitute Eq. (33) in Eq. (13) we get

$$
P^{(1)}=\int_{V} d V \partial_{1}\left(\Pi^{(1) 1}(r, \theta, \phi)\right)=\int_{S} d S_{1} \Pi^{(1) 1}(r, \theta, \phi)=0 .
$$

By the same method we obtain

$$
\Pi^{(2) 1}=0, \quad P^{(2)}=0, \quad \Pi^{(3) 1}=0, \quad P^{(3)}=0 .
$$

The results of Eqs. (34) and (35) are expected results since the space-time given by Eq. (22) is a spherical symmetric static space-time. Therefore, the spatial momentum associated with any static solution is identically vanishing [58].

We have used Eqs. (19) and (6) in Eq. (20) to calculate the components of the angularmomentum. Finally we get

$$
\begin{aligned}
& M^{(1)(2)}=e^{(1)}{ }_{a} e^{(2)}{ }_{b} M^{a b}=M^{(1)(3)}=e^{(1)}{ }_{a} e^{(3)}{ }_{b} M^{a b}=M^{(2)(3)}=e^{(2)}{ }_{a} e^{(3)}{ }_{b} M^{a b}=0 \\
& M^{(0)(1)}=e^{(0)} e^{(1)}{ }_{b} M^{a b}=-\frac{\sqrt{r} A^{2} \sin ^{2} \theta \cos \phi\{r-\sqrt{r} B\}}{4 \pi B}, \\
& M^{(0)(2)}=e^{(0)}{ }_{a} e^{(2)}{ }_{b} M^{a b}=-\frac{\sqrt{r} A^{2} \sin ^{2} \theta \sin \phi\{r-\sqrt{r} B\}}{4 \pi B}, \\
& M^{(0)(3)}=e^{(0)}{ }_{a} e^{(3)}{ }_{b} M^{a b}=-\frac{\sqrt{r} A^{2} \sin \theta \cos \theta\{r-\sqrt{r} B\}}{4 \pi B} .
\end{aligned}
$$

Using Eq. (36) in (20) we get

$$
\begin{aligned}
L^{(0)(1)}= & \int_{0}^{\pi} \int_{0}^{2 \pi} \int_{0}^{\infty} d \theta d \phi d r M^{(0)(1)}(r, \theta, \phi)=0, \\
& \text { by the same method we can get } \\
L^{(0)(2)}= & L^{(0)(3)}=L^{(1)(2)}=L^{(1)(3)}=L^{(2)(3)}=0 .
\end{aligned}
$$

It is of interest to note that the vanishing of $L^{(0)(1)}, L^{(0)(2)}$ is due to the appearance of terms like $\sin \phi$ and $\cos \phi$ while the vanishing of $L^{(0)(3)}$ is due to the appearance of term like $\sin \theta \cos \theta$.

Repeating the same calculations to the second tetrad given by Eq. (23) and write the necessary components of the torsion tensor and the vector field $T^{(a)}$, we get

$$
T_{01}^{(0)}=\frac{A^{\prime}}{A}, \quad T_{12}^{(2)}=T_{13}^{(3)}=\frac{-1}{r}, \quad T_{23}^{(3)}=-\cot \theta,
$$


and the only non-vanishing components of the tensor $T^{(a)}$ are given by

$$
T^{(1)}=\frac{B^{2}\left\{2 A+r A^{\prime}\right\}}{r^{2} A}, \quad T^{(2)}=\frac{\cot \theta}{r^{2}} .
$$

The only required component of $\Sigma^{\mu \nu \lambda}$ needed to calculate the energy is

$$
\Sigma^{(0) 01}=\frac{\sqrt{r} B \sin \theta}{4 \pi} .
$$

Further substituting Eq. (40) into Eq. (13) we obtain

$$
P^{(0)}=E=-\oint_{S \rightarrow \infty} d S_{k} \Pi^{(0) k}=-\frac{1}{4 \pi} \oint_{S \rightarrow \infty} d S_{k} e \Sigma^{(0) 0 k}=\sqrt{r} B .
$$

By the same method used for the first tetrad given by Eq. (22) we find that the momentum and angular-momentum associated with the second tetrad field given by Eq. (23) have the form

$$
\begin{aligned}
& \Pi^{(1) 1}=0, \quad P^{(1)}=\int_{V} d V \partial_{1}\left(\Pi^{(1) 1}(r, \theta, \phi)\right)=\int_{S} d S_{1} \Pi^{(1) 1}(r, \theta, \phi)=0, \\
& \Pi^{(2) 1}=0, \quad P^{(2)}=0, \quad \Pi^{(3) 1}=0, \quad P^{(3)}=0,
\end{aligned}
$$

$$
\begin{aligned}
& M^{(1)(2)}=e^{(1)}{ }_{a} e^{(2)}{ }_{b} M^{a b}=M^{(1)(3)}=e^{(1)}{ }_{a} e^{(3)}{ }_{b} M^{a b}=M^{(2)(3)}=e^{(2)}{ }_{a} e^{(3)}{ }_{b} M^{a b}=M^{(0)(3)}=e^{(0)}{ }_{a} e^{(3)}{ }_{b} M^{a b}=0 \\
& M^{(0)(1)}=e^{(0)}{ }_{a} e^{(1)}{ }_{b} M^{a b}=\frac{r A^{2} \sin \theta}{4 \pi}, \quad M^{(0)(2)}=e^{(0)}{ }_{a} e^{(2)}{ }_{b} M^{a b}=\frac{r^{3 / 2} A^{2} \cos \theta}{8 \pi B} .
\end{aligned}
$$

Using Eq. (43) in (20) we get

$L^{(0)(1)}=\int_{0}^{\pi} \int_{0}^{2 \pi} \int_{0}^{\infty} d \theta d \phi d r M^{(0)(1)}(r, \theta, \phi)=-\int_{0}^{\infty} \frac{r^{3 / 2} A^{2}}{B} d r \neq 0, \quad$ by the same method we can obtain $L^{(0)(2)}=L^{(0)(3)}=L^{(1)(2)}=L^{(1)(3)}=L^{(2)(3)}=0$.

It is of interest to note that the vanishing of $L^{(0)(2)}$ is due to the appearance of terms like $\cos \theta$. Here we obtain the component of the angular momentum $L^{(0)(1)} \neq 0$ contradict what is well know that the angular momentum of a spherically symmetric stationary spacetime is vanishing [58].

Let us gave a specific value for the two unknown functions $A$ and $B$ to have the form

$$
A=\sqrt{1-\frac{2 M}{r}}, \quad \text { and } \quad B=\sqrt{\frac{\left(1-\frac{3 M}{2 r}\right)}{\left(1-\frac{2 M}{r}\right)\left(1-\frac{\lambda_{0}}{r}\right)}} .
$$

This is a solution obtained before by Casadio et al. [59] in search for new brane world black holes and by Germani et al. [60] as a possible external metric of a homogeneous star on the 
brane. From Eq. (4) using the tetrad field (22) and Eq. (45) we get the associated metric in the form

$$
d s^{2}=-\left(1-\frac{2 M}{r}\right) d t^{2}+\frac{\left(1-\frac{3 M}{2 r}\right)}{\left(1-\frac{2 M}{r}\right)\left(1-\frac{\lambda_{0}}{r}\right)} d r^{2}+r^{2}\left(d \theta^{2}+\sin ^{2} \theta d \phi^{2}\right) .
$$

The Schwarzschild metric is restored in the special case $\lambda_{0}=\frac{3 M}{2}$. In the case when $\lambda_{0}>2 M$ the metric of Eq. (46) describes a symmetric traversable wormhole [61]. If we apply Eq. (13) to the tetrad given by Eq. (22) using (45) we get the energy to has the form

$$
P^{(0)} \cong \int_{r \rightarrow \infty} d \theta d \phi \frac{1}{16 \pi} \sin \theta\left(M+2 \lambda_{0}\right)=\frac{M}{4}+\frac{\lambda_{0}}{2}
$$

and when $\lambda_{0}=\frac{3 M}{2}$ we get

$$
P^{(0)} \cong M,
$$

which is the ADM [58]. The energy associated with the second tetrad given by Eq. (23) using Eq. (45) is given by

$$
P^{(0)} \cong-r+\frac{M}{4}+\frac{\lambda_{0}}{2} !
$$

which is different from the energy given by Eq. (47).

As we see from Eqs. (47) and (49) that the energy associated with the two tetrad field given be Eqs. (22) and (23) are different in spite that they reproduce the same space-time as given by Eq. (46). Definition of energy as given by Eq. (13) depends mainly on the definition of torsion and the components of the torsion of both tetrad fields given by Eqs. (26) and (38) which are different. The flatness condition given by Eq. (25) of both tetrad fields are satisfied when $A=1$ and $B=1$. The components of the torsion associated with the two tetrad fields (22) and (23) are now have the following form after using Eq. (45). For the tetrad (22) we have

$T_{01}^{(0)}=\frac{M}{r(r-2 M)}, \quad T_{12}^{(2)}=\frac{\sqrt{r(r-2 M)\left(r-\lambda_{0}\right)(4 r-6 M)}-2(r-2 M)\left(r-\lambda_{0}\right)}{2 r(r-2 M)\left(r-\lambda_{0}\right)}=T^{(3)}{ }_{13}$,

and for the tetrad (23) we have

$$
T^{(0)}{ }_{01}=\frac{M}{r(r-2 M)}, \quad T_{12}^{(2)}=-\frac{1}{r}=T_{13}^{(3)}, \quad T_{23}^{(3)}=-\cot \theta .
$$

The space-time (46) is a flat space-time when $M=0$ and $\lambda_{0}=0$ in this case the components of the torsion tensor of the first tetrad are vanishing identically, $T^{(a)}{ }_{\mu \nu}=0$ satisfying the flatness condition given by Eq. (25). The components of torsion given by Eq. (51) of the second tetrad when $M=0$ and $\lambda_{0}=0$ do not vanishing identically contradict the flatness condition given by Eq. (25). Therefore, in this case we are going to use the regularized expression for the gravitational energy-momentum. 


\section{Regularized expression for the gravitational energy-momentum and localization of energy}

An important property of the tetrad fields that satisfy the condition of Eq. (25) is that in the flat space-time limit $e^{a}{ }_{\mu}(t, x, y, z)=\delta^{a}{ }_{\mu}$, and therefore the torsion $T^{\lambda}{ }_{\mu \nu}=0$. Hence for the flat space-time it is normally to consider a set of tetrad fields such that $T^{\lambda}{ }_{\mu \nu}=0$ in any coordinate system. However, in general an arbitrary set of tetrad fields that yields the metric tensor for the asymptotically flat space-time does not satisfy the asymptotic condition given by (25). Moreover for such tetrad fields the torsion $T_{\mu \nu}^{\lambda} \neq 0$ for the flat space-time [62]. It might be argued, therefore, that the expression for the gravitational energy-momentum (13) is restricted to particular class of tetrad fields, namely, to the class of frames such that $T^{\lambda}{ }_{\mu \nu}=0$ if $E_{a}{ }^{\mu}$ represents the flat space-time tetrad field [62]. To explain this, let us calculate the flat space-time of the tetrad field of Eq. (23) using (46) which is given by

$$
\left(E_{2 a}{ }^{\mu}\right)=\left(\begin{array}{cccc}
1 & 0 & 0 & 0 \\
0 & 1 & 0 & 0 \\
0 & 0 & \frac{1}{r} & 0 \\
0 & 0 & 0 & \frac{1}{r \sin \theta}
\end{array}\right) .
$$

Expression (52) yields the following non-vanishing torsion components:

$$
T_{12}^{(2)}=-\frac{1}{r}=T_{13}^{(3)}, \quad T_{23}^{(3)}=-\cot \theta .
$$

The tetrad field (52) when written in the Cartesian coordinate will have the form

$$
\left(E_{2 a}^{\mu}(t, x, y, z)\right)=\left(\begin{array}{cccc}
1 & 0 & 0 & 0 \\
0 & \frac{x}{r} & \frac{y}{r} & \frac{z}{r} \\
0 & \frac{x z}{r \sqrt{x^{2}+y^{2}}} & \frac{y z}{r \sqrt{x^{2}+y^{2}}} & -\frac{\sqrt{x^{2}+y^{2}}}{r} \\
0 & -\frac{y}{\sqrt{x^{2}+y^{2}}} & \frac{x}{\sqrt{x^{2}+y^{2}}} & 0
\end{array}\right) .
$$

In view of the geometric structure of (54), we see that, Eq. (23) does not display the asymptotic behavior required by Eq. (25). Moreover, in general the tetrad field (54) is adapted to accelerated observers [28, 30, 62]. To explain this, let us consider a boost in the $\mathrm{x}$-direction of Eq. (54). We find

$$
\left(E_{2 a}^{\mu}(t, x, y, z)\right)=\left(\begin{array}{cccc}
\gamma & v \gamma & 0 & 0 \\
\frac{v \gamma x}{r} & \frac{\gamma x}{r} & \frac{y}{r} & \frac{z}{r} \\
\frac{v \gamma x z}{r \sqrt{x^{2}+y^{2}}} & \frac{\gamma x z}{r \sqrt{x^{2}+y^{2}}} & \frac{y z}{r \sqrt{x^{2}+y^{2}}} & \frac{-\sqrt{x^{2}+y^{2}}}{r} \\
\frac{-v \gamma y}{\sqrt{x^{2}+y^{2}}} & \frac{-\gamma y}{\sqrt{x^{2}+y^{2}}} & \frac{x}{\sqrt{x^{2}+y^{2}}} & 0
\end{array}\right),
$$


where $v$ is the speed of the observer and $\gamma=\frac{1}{\sqrt{1-v^{2}}}$. For a static object in a space-time whose four-velocity is given by $u^{\mu}=(1,0,0,0)$ we may compute its frame components $u^{a}=e^{a}{ }_{\mu} u^{\mu}=\left(\gamma, \frac{v \gamma x}{r}, \frac{v \gamma x z}{r \sqrt{x^{2}+y^{2}}}, \frac{-v \gamma y}{\sqrt{x^{2}+y^{2}}}\right)$. It can be shown that along an observer's trajectory whose velocity is determined by $u^{a}$ the quantities

$$
\phi_{(j)}^{(k)}=u^{i}\left(E_{2}{ }^{(k)}{ }_{m} \partial_{i} E_{2(j)}{ }^{m}\right)
$$

constructed out from (55) are non vanishing. This fact indicates that along the observer's path the spatial axis $E_{2(a)}{ }^{\mu}$ rotate $[28,62]$. In spite of the above problems discussed for the tetrad field of Eq. (23) it yields a satisfactory value for the total gravitational energymomentum, as we will discussed.

In Eq. (13) it is implicitly assumed that the reference space is determined by a set of tetrad fields $e^{a}{ }_{\mu}$ for flat space-time such that the condition $T^{a}{ }_{\mu \nu}=0$ is satisfied. However, in general there exist flat space-time tetrad fields for which $T_{\mu \nu}^{a} \neq 0$. In this case Eq. (13) may be generalized $[28,62]$ by adding a suitable reference space subtraction term, exactly like in the Brown-York formalism [63, 64].

We will denote $T^{a}{ }_{\mu \nu}(E)=\partial_{\mu} E^{a}{ }_{\nu}-\partial_{\nu} E^{a}{ }_{\mu}$ and $\Pi^{a j}(E)$ as the expression of $\Pi^{a j}$ constructed out of the flat tetrad $E^{a}{ }_{\mu}$. The regularized form of the gravitational energy-momentum $P^{a}$ is defined by $[28,62]$

$$
P^{a}=-\int_{V} d^{3} x \partial_{k}\left[\Pi^{a k}(e)-\Pi^{a k}(E)\right] .
$$

This condition guarantees that the energy-momentum of the flat space-time always vanishes. The reference space-time is determined by tetrad fields $E^{a}{ }_{\mu}$, obtained from $e^{a}{ }_{\mu}$ by requiring the vanishing of the physical parameters like mass, angular-momentum, etc. Assuming that the space-time is asymptotically flat then Eq. (57) can have the form [28, 62]

$$
P^{a}=-\oint_{S \rightarrow \infty} d S_{k}\left[\Pi^{a k}(e)-\Pi^{a k}(E)\right],
$$

where the surface $S$ is established at spacelike infinity. Eq. (58) transforms as a vector under the global $\mathrm{SO}(3,1)$ group [30]. Now we are in a position to proof that the tetrad field (23) yields a satisfactory value for the total gravitational energy-momentum.

We will integrate Eq. (58) over a surface of constant radius $x^{1}=r$ and require $r \rightarrow \infty$. Therefore, the index $k$ in (58) takes the value $k=1$. We need to calculate the quantity

$$
\Sigma^{(0) 01}=e^{(0)}{ }_{0} \Sigma^{001}=\frac{1}{2} e^{(0)}{ }_{0}\left(T^{001}-g^{00} T^{1}\right) .
$$

Evaluate the above equation we find

$$
\Pi^{(0) 1}(e)=\frac{-1}{4 \pi} e \Sigma^{(0) 01}=-\frac{1}{4 \pi} \frac{\sin \theta \sqrt{r(r-2 M)\left(2 r-2 \lambda_{0}\right)}}{\sqrt{2 r-3 M}} \cong \frac{-\sin \theta\left(4 r-M-2 \lambda_{0}\right)}{16 \pi}
$$

and the expression of $\Pi^{(0) 1}(E)$ is obtained by just making $M=0$ and $\lambda_{0}=0$ in Eq.(59), it is given by

$$
\Pi^{(0) 1}(E)=\frac{-1}{4 \pi} r \sin (\theta)
$$


Thus the gravitational energy of the tetrad field of Eq. (23) is given by

$$
P^{0} \cong \int_{r \rightarrow \infty} d \theta d \phi \frac{1}{16 \pi} \sin \theta\left\{\left(-4 r+M+2 \lambda_{0}\right)+4 r\right\}=\frac{M}{4}+\frac{\lambda_{0}}{2}
$$

which is exactly the energy of the first tetrad (22) as given by Eq. (47).

\section{Teleparallel Killing Vectors of the Bran-world Spacetimes}

In this section we are going to calculate the Killing vectors of the two tetrad space-times, given by Eqs (22) and (23) to make the picture more clear about the different results we

obtained for the energy and angular-momentum. Using the teleparallel Lie derivatives of a covariant tensor of rank 2 established in [65] which is defined as

$$
\left(\mathcal{L}^{T}{ }_{\xi} g\right)_{\mu \nu} \stackrel{\text { def. }}{=} g_{\mu \nu, \rho} \xi^{\rho}+g_{\rho \nu} \xi^{\rho}, \mu+g_{\mu \rho} \xi^{\rho}, \nu+\xi^{\rho}\left(g_{\epsilon \nu} T_{\mu \rho}^{\epsilon}+g_{\epsilon \mu} T_{\nu \rho}^{\epsilon}\right)
$$

where, denote the ordinary derivative, $\xi$ is a vector field and $T^{\epsilon}{ }_{\mu \rho}$ is the torsion tensor defined by Eq. (7). The teleparallel Killing equations is defined as [65]

$$
\left(\mathcal{L}^{T} \xi\right)_{\mu \nu} \stackrel{\text { def. }}{=} 0
$$

Apply Eq. (63) to Eq. (22) we get

$$
\begin{aligned}
2 A^{\prime}(r) \xi^{1}(r, \theta, \phi, t)+A(r) \xi^{0},{ }_{0}(r, \theta, \phi, t) & =0, \\
A^{2}(r) B^{2}(r) \xi_{,{ }_{1}}(r, \theta, \phi, t)-r \xi_{, 0}^{1}(r, \theta, \phi, t)-A(r) B^{2}(r) A^{\prime}(r) \xi^{0}(r, \theta, \phi, t) & =0, \\
A^{2}(r) \xi^{0},{ }_{2}(r, \theta, \phi, t)-r^{2} \xi^{2},{ }_{0}(r, \theta, \phi, t) & =0, \\
A^{2}(r) \xi^{0},{ }_{3}(r, \theta, \phi, t)-r^{2} \sin ^{2} \theta \xi^{3},{ }_{0}(r, \theta, \phi, t) & =0, \\
\left(2 r B^{\prime}(r)-B(r)\right) \xi^{1}(r, \theta, \phi, t)-2 r B(r) \xi^{1},{ }_{1}(r, \theta, \phi, t) & =0, \\
B(r) \xi^{2}(r, \theta, \phi, t)(B(r)-\sqrt{r})+r B^{2}(r) \xi^{2},{ }_{1}(r, \theta, \phi, t)+\xi^{1},{ }_{2}(r, \theta, \phi, t) & =0, \\
B(r) \sin ^{2} \theta \xi^{3}(r, \theta, \phi, t)(B(r)-\sqrt{r})+\xi^{1},{ }_{3}(r, \theta, \phi, t)+r B^{2}(r) \sin ^{2} \theta \xi^{3},{ }_{1}(r, \theta, \phi, t) & =0, \\
r B(r) \xi^{2},{ }_{2}(r, \theta, \phi, t)+\sqrt{r} \xi^{1}(r, \theta, \phi, t) & =0, \\
\xi^{2},{ }_{3}(r, \theta, \phi, t)+\sin ^{2} \theta \xi^{3},{ }_{2}(r, \theta, \phi, t) & =0, \\
r B(r) \cos \theta \xi^{2}(r, \theta, \phi, t)+r B(r) \sin \theta \xi^{3},{ }_{3}(r, \theta, \phi, t)+\sqrt{r} \sin \theta \xi^{1}(r, \theta, \phi, t) & =0,
\end{aligned}
$$

where $\xi^{\alpha}{ }_{, a}=\frac{\partial \xi^{\alpha}}{\partial x^{a}}$. From the first and fifth equations of Eq. (64) we get

$$
\xi^{1}(r, \theta, \phi, t)=\frac{B(r) F(\theta, \phi, t)}{\sqrt{r}}, \quad \xi^{0}(r, \theta, \phi, t)=-\int \frac{2 A^{\prime}(r) B(r) F(\theta, \phi, t)}{A(r) \sqrt{r}} d t+F_{1}(r, \theta, \phi),
$$


using Eq. (65) in the sixth and seventh equations of Eq. (64) we get

$$
\begin{aligned}
& \xi^{2}(r, \theta, \phi, t)=e^{\int\left(\frac{\sqrt{r}-B(r)}{r B(r)}\right) d r}\left\{-\int\left(\frac{F_{2}(\theta, \phi, t) e^{\int\left(\frac{\sqrt{r}-B(r)}{r B(r)}\right) d r}}{r^{3 / 2} B(r)}\right) d r+F_{2}(\theta, \phi, t)\right\} \\
& \xi^{3}(r, \theta, \phi, t)=e^{\int\left(\frac{\sqrt{r}-B(r)}{r B(r)}\right) d r}\left\{-2 \int\left(\frac{F_{3}(\theta, \phi, t) e^{\int\left(-\frac{\sqrt{r}-B(r)}{r B(r)}\right) d r}}{r^{3 / 2} B(r)(\cos 2 \theta-1)}\right) d r+F_{3}(\theta, \phi, t)\right\}
\end{aligned}
$$

where $F(\theta, \phi, t), F_{1}(r, \theta, \phi), F_{2}(\theta, \phi, t)$ and $F_{3}(\theta, \phi, t)$ are arbitrary functions to be determined from the remaining equations of Eq. (64). Using Eqs. (65) and (66) in the remaining equations of Eq. (64) we finally get

$$
\begin{aligned}
& \xi^{0}(r, \theta, \phi, t)=C_{0} A(r), \quad \xi^{1}(r, \theta, \phi, t)=0, \quad \xi^{2}(r, \theta, \phi, t)=\frac{e^{\int \frac{1}{\sqrt{r} B(r)} d r}}{r}\left(C_{1} \sin \phi+C_{2} \cos \phi\right), \\
& \xi^{3}(r, \theta, \phi, t)=\frac{e^{\int \frac{1}{\sqrt{r} B(r)} d r}}{r \sin \theta}\left(C_{1} \cos \theta \cos \phi-C_{2} \cos \theta \sin \phi+C_{3} \sin \theta\right),
\end{aligned}
$$

where $C_{0}, C_{1}, C_{2}$, and $C_{3}$ are four constants of integration. Thus

$$
\begin{aligned}
\xi= & \left(C_{0} A(r) \frac{\partial}{\partial t}+\frac{e^{\int \frac{1}{\sqrt{r} B(r)} d r}}{r}\left\{C_{1} \sin \phi+C_{2} \cos \phi\right\} \frac{\partial}{\partial \theta}\right. \\
& \left.+\frac{e^{\int \frac{1}{\sqrt{r} B(r)} d r}}{r \sin \theta}\left\{C_{1} \cos \theta \cos \phi-C_{2} \cos \theta \sin \phi+C_{3} \sin \theta\right\} \frac{\partial}{\partial \phi}\right) .
\end{aligned}
$$

Eq. (68) gives the 4 Killing vector of the spacetime given by Eq. (22) in the context of the TEGR as

$$
\begin{aligned}
& \xi_{(1)}=A(r) \frac{\partial}{\partial t}, \\
& \xi_{(2)}=\frac{e^{\int \frac{1}{\sqrt{r} B(r)} d r}}{r \sin \theta}\left(\sin \theta \cos \phi \frac{\partial}{\partial \theta}-\cos \theta \sin \phi \frac{\partial}{\partial \phi}\right), \\
& \xi_{(3)}=\frac{e^{\int \frac{1}{\sqrt{r} B(r)} d r}}{r \sin \theta}\left(\sin \theta \sin \phi \frac{\partial}{\partial \theta}+\cos \theta \cos \phi \frac{\partial}{\partial \phi}\right), \\
& \xi_{(4)}=\frac{e^{\int \frac{1}{\sqrt{r} B(r)} d r}}{r} \frac{\partial}{\partial \phi} .
\end{aligned}
$$

Now turn our attention to the derivation of the killing vector of the second tetrad given by Eq. (23). Apply Eq. (63) to Eq. (23) we get

$$
\begin{aligned}
2 A^{\prime}(r) \xi^{1}(r, \theta, \phi, t)+A(r) \xi^{0}{ }_{0}(r, \theta, \phi, t) & =0, \\
A^{2}(r) B^{2}(r) \xi_{,{ }_{1}}^{0}(r, \theta, \phi, t)-r \xi_{, 0}^{1}(r, \theta, \phi, t)-A(r) B^{2}(r) A^{\prime}(r) \xi^{0}(r, \theta, \phi, t) & =0, \\
A^{2}(R) \xi^{0}{ }_{2}(r, \theta, \phi, t)-r^{2} \xi^{2}{ }_{0}(r, \theta, \phi, t) & =0,
\end{aligned}
$$




$$
\begin{aligned}
A^{2}(r) \xi^{0},{ }_{3}(r, \theta, \phi, t)-r^{2} \sin ^{2} \theta \xi^{3},{ }_{0}(r, \theta, \phi, t) & =0, \\
\left(2 r B^{\prime}(r)-B(r)\right) \xi^{1}(r, \theta, \phi, t)-2 r B(r) \xi^{1},{ }_{1}(r, \theta, \phi, t) & =0, \\
B^{2}(r) \xi^{2}(r, \theta, \phi, t)+r B^{2}(r) \xi^{2},{ }_{1}(r, \theta, \phi, t)+\xi^{1},{ }_{2}(r, \theta, \phi, t) & =0, \\
B^{2}(r) \sin ^{2} \theta \xi^{3}(r, \theta, \phi, t)+\xi^{1},{ }_{3}(r, \theta, \phi, t)+r B^{2}(r) \sin ^{2} \theta \xi^{3},{ }_{1}(r, \theta, \phi, t) & =0, \\
\xi^{2},{ }_{2}(r, \theta, \phi, t) & =0, \\
\xi^{2},{ }_{3}(r, \theta, \phi, t)-\sin ^{2} \theta \xi^{3},{ }_{2}(r, \theta, \phi, t)+\xi^{3}(r, \theta, \phi, t) \sin \theta \cos \theta & =0, \\
\xi^{3},{ }_{3}(r, \theta, \phi, t) & =0 .
\end{aligned}
$$

From the first, fifth, eighth and tenth equations of Eq. (70) we get

$$
\begin{aligned}
& \xi^{1}(r, \theta, \phi, t)=\frac{B(r)}{\sqrt{r}} F_{4}(\theta, \phi, t), \quad \xi^{0}(r, \theta, \phi, t)=-\int \frac{2 A^{\prime}(r) B(r)}{\sqrt{r} A(r)} F_{4}(\theta, \phi, t) d t+F_{5}(r, \theta, \phi), \\
& \xi^{2}(r, \theta, \phi, t)=F_{6}(r, \phi, t), \quad \xi^{3}(r, \theta, \phi, t)=F_{7}(r, \theta, t),
\end{aligned}
$$

where $F_{4}(\theta, \phi, t), F_{5}(r, \theta, \phi), F_{6}(r, \phi, t)$ and $F_{7}(r, \theta, t)$ are arbitrary functions to be determined from the remaining equations of Eq. (70). Using Eq. (71) in the remaining equations of Eq. (70) we finally get

$$
\xi^{0}(r, \theta, \phi, t)=0, \quad \xi^{1}(r, \theta, \phi, t)=0, \quad \xi^{2}(r, \theta, \phi, t)=\frac{C_{4}}{r}, \quad \xi^{3}(r, \theta, \phi, t)=\frac{C_{5}}{r \sin \theta},
$$

where $C_{4}$ and $C_{5}$ are two constants of integration. Thus

$$
\xi=\left(\frac{C_{4}}{r} \frac{\partial}{\partial \theta}+\frac{C_{5}}{r \sin \theta} \frac{\partial}{\partial \phi}\right) .
$$

Eq. (73) gives the 2 Killing vector of the space-time given by Eq. (23) in the context of the TEGR as

$$
\xi_{(1)}=\frac{1}{r} \frac{\partial}{\partial \theta}, \quad \xi_{(2)}=\frac{1}{r \sin \theta} \frac{\partial}{\partial \phi} .
$$

It is well known that Minkowski spacetime has the Poincaré symmetry algebra with the 10 generators $[66,67]$

$$
\begin{aligned}
& \xi_{0}=\frac{\partial}{\partial t}, \quad \xi_{1}=\cos \phi \frac{\partial}{\partial \theta}-\cot \theta \sin \phi \frac{\partial}{\partial \phi} \\
& \xi_{2}=\sin \phi \frac{\partial}{\partial \theta}+\cot \theta \cos \phi \frac{\partial}{\partial \phi}, \quad \xi_{3}=\frac{\partial}{\partial \phi} \\
& \xi_{4}=\sin \theta \cos \phi \frac{\partial}{\partial r}+\frac{\cos \theta \cos \phi}{r} \frac{\partial}{\partial \theta}-\frac{\csc \theta \sin \phi}{r} \frac{\partial}{\partial \phi} \\
& \xi_{5}=\sin \theta \sin \phi \frac{\partial}{\partial r}+\frac{\cos \theta \sin \phi}{r} \frac{\partial}{\partial \theta}+\frac{\csc \theta \cos \phi}{r} \frac{\partial}{\partial \phi} \\
& \xi_{6}=\cos \theta \frac{\partial}{\partial r}-\frac{\sin \theta}{r} \frac{\partial}{\partial \theta},
\end{aligned}
$$




$$
\begin{aligned}
& \xi_{7}=r \sin \theta \cos \phi \frac{\partial}{\partial t}+t\left\{\sin \theta \cos \phi \frac{\partial}{\partial r}+\frac{\cos \theta \cos \phi}{r} \frac{\partial}{\partial \theta}-\frac{\csc \theta \sin \phi}{r} \frac{\partial}{\partial \phi}\right\}, \\
& \xi_{8}=r \sin \theta \sin \phi \frac{\partial}{\partial t}+t\left\{\sin \theta \sin \phi \frac{\partial}{\partial r}+\frac{\cos \theta \sin \phi}{r} \frac{\partial}{\partial \theta}+\frac{\csc \theta \cos \phi}{r} \frac{\partial}{\partial \phi}\right\}, \\
& \xi_{9}=r \cos \theta \frac{\partial}{\partial t}+t\left\{\cos \theta \frac{\partial}{\partial r}-\frac{\sin \theta}{r} \frac{\partial}{\partial \theta}\right\},
\end{aligned}
$$

where the speed of light $c=1$ and $\xi_{0}, \xi_{4}, \xi_{5}$ and $\xi_{6}$ are the spacetime translations which provide the laws of conservation of energy and linear momentum, $\xi_{1}, \xi_{2}$ and $\xi_{3}$ are the rotations which provide the laws of conservation of angular momentum and $\xi_{7}, \xi_{8}$ and $\xi_{9}$ are the Lorentz transformations which provide the laws of conservation of spin and angular momentum via Noether's theorem [68]. For a spherically symmetric space-time only the 4 generators $\xi_{0}, \xi_{1}, \xi_{2}$ and $\xi_{3}$ apply, yielding only conservation of energy and angular momentum. The generator $\xi_{4}, \xi_{5}, \xi_{6}, \xi_{7}, \xi_{8}$ and $\xi_{9}$ yielding conservation of linear momentum and spin angular momentum are lost for this space-time.

Let us compare our results with the first four equations of Eq. (75). For the tetrad field given by Eq. (22) we get the 4 Killing vectors to have the form given by Eq. (69). This Killing vectors are in consistence with the first 4 Killing vectors of Eq. (75) when $A(r)=1$ and $B(r)=\sqrt{r}$. Energy, irreducible mass, momentum and angular momentum associated with the tetrad given by Eq. (22) are satisfactory and in consistence with the previous results [55]. So this tetrad has no problem either for the calculations of energy, irreducible mass, momentum and angular momentum or for the associated Killing vectors which given by Eq. (69).

The tetrad given by Eq. (23) has many problems: First the energy is not equal the ADM. Also the irreducible mass is effected by the horizon and some components of the angular momentum are not vanishing. This may be due to the fact that the Killing vector associated with tetrad (23) is not in agreement with that given by Eq. (75). Since the first four equations of Eq. (75) are the responsible for the conservation of energy and angular momentum and these equations are disappear from tetrad (23) as is clear from by Eq. (74).

\section{Main results and Discussion}

The main results of this paper can be summarized as follow:

- Two different tetrad fields are used. These tetrads are related by a local Lorentz transformation which keeps spherical symmetry, i.e., the tetrad (22) can be written in terms of the tetrad (23) using the following local Lorentz transformation

$$
\left(e_{1 a}{ }^{\mu}\right)=\Lambda_{\nu}{ }^{\mu}\left(e_{2 a}{ }^{\nu}\right), \text { where } \Lambda_{\nu}{ }^{\mu}=\left(\begin{array}{ccccc}
1 & 0 & 0 & 0 \\
0 & \sin \theta \cos \phi & \cos \theta \cos \phi & -\sin \phi \\
0 & \sin \theta \sin \phi & \cos \theta \sin \phi & \cos \phi \\
0 & \cos \theta & -\sin \theta & 0
\end{array}\right)
$$


The space-time associated with these tetrad fields is given by Eq. (24).

- The energy of these tetrad fields are calculated using the gravitational energy-momentum tensor, which is a coordinate independent [30]. One of this tetrad field given by Eq. (22) gives a satisfactory results for the energy, while the other which is given by Eq. (23) its associated energy depends on the radial coordinate.

- Calculations of the torsion components associated with these two tetrad fields are given. From these calculations we show that the torsion components of each tetrad field are different. This may gave an indication why the energy of the two tetrad fields is different.

- We use the regularized expression of the gravitational energy-momentum tensor to calculate the energy associated with the second tetrad field given by Eq. (23).

- After using the regularized expression of the gravitational energy-momentum tensor we show that the energy associated with the two tetrad fields is equal.

- Using the definition of the energy and the angular momentum given by Eqs. (13) and (20) we show by explicit calculations that the angular momentum depends on the choice of the frame used.

- The calculations of the irreducible mass is given within the external horizons. From this calculations we show that the external horizons do not play any role on the energy

- To make the picture more clear we have calculated the Killing vectors associated with these tetrad fields using the definition of the Lie derivative of a second rank tensor in the framework of TEGR [65]. We have shown by explicit calculations that the tetrad which show consistence results of energy, irreducible mass, momentum and angular momentum continue to show consistency for the Killing vector with GR [66]. The other tetrad fields also continue to give results of Killing vectors in contradiction with that of GR. This contradiction clear why the results of energy, irreducible mass, momentum and angular momentum are not in consistence. 


\section{References}

[1] T. Kaluza, Sitzungseber. Press. Akad. Wiss. Phys. Math. Klasse K1 (1920), 966; O. Klein, 1Z. Phys. 37 (1926), 895.

[2] P. Hor̃ava and E. Witten, Nucl. Phys. B460, (1996), 506; B475 (1996) 94.

[3] L. Randall and R. Sundrum, Phys. Rev. Lett. 83 (1999) 3370; 4690.

[4] T. Shiromizu, K. Maeda and M. Sasaki, Phys. Rev. D62 (2000), 024012.

[5] K.A. Bronnikov, V.N. Melnikov and H. Dehnen, Phys. Rev. D68 (2003), 024025.

[6] K.A. Bronnikov, G. Clément, C.P. Constantinidis and J.C. Favris, Phys. Lett.A243 (1998), 121; Grav. Cos. 4 (1998), 128.

[7] T.W.B. Kibble, J.Math. Phys. 2 (1961) 212.

[8] F.W. Hehl, P. Von der Heyde, D. Kerlick and J. Nester, Rev. Mod. Phys. 48 (1976), 393.

[9] F.W. Hehl, in: General Relativity and Gravitation- One Hundred Years after the birth of Albert Einstein, ed. A. Held (Plenum, New York, 1980) Vol 1.

[10] K. Hayashi, and T. Shirafuji, Prog. Theor. Phys. 64 (1980), 866, 883, 1435, 2222; 65, 525 .

[11] M. Blagojević and I.A. Nikolić Phys. Rev. D62 (2000), 024021.

[12] F.W. Hehl, J. Nitsch and P. von der Heyde, in General Relativity and Gravitation, A. Held, ed. (Plenum Press, New York) (1980).

[13] F.W. Hehl, J.D. MacCrea, E.W. Mielke and Y. Ne'eman, Phys. Rep. 258 (1995), 1.

[14] K. Hayashi and T. Shirafuji, Phys. Rev. D19 (1979), 3524.

[15] J. Nitsch, in: Cosmology and Gravitation: Spin, Torsion, Rotation and Supergravity, eds. Bergman and V. de Sabbata (Plenum, New York, 1980).

[16] P.G. Bergmann and R. Thomson, Phys. Rev. 89 (1953), 401.

[17] L.D. Landau and E.M. Lifshitz, The Classical Theory of Fields (Pergamon Press, Oxford, 1980).

[18] C. Pellegrini and J. Plebanski, Mat. Fys. Scr. Dan. Vid. Selsk. 2 (1963), no.3.

[19] C. Møller, (1961) Ann. of Phys. 12 (1961), 118.

[20] C. Møller, "Tetrad fields and conservation laws in general relativity" in Proc. International School of Physics "Enrico Fermi" ed. C. Møller, (Academic Press, London, 1962).

[21] C. Møller, Mat. Fys. Medd. Dan. Vid. Selsk. 1 (1961), 10. 
[22] C. Møller, Nucl. Phys. 57 (1964), 330.

[23] K. Hayashi and T. Nakano, Prog. Theor. Phys. 38 (1967), 491.

[24] W. Kopzyński, J. Phys. A15 (1982), 493.

[25] C.C. Chang, J.M. Nester and C.M. Chen, Phys. Rev. Lett. 83 (1999), 1897; R.S. Tung and J.M. Nester, Phys. Rev. $D 60$ (1999) 021501; J.M. Nester and H.J. Yo Chin, J. Phys. 37 (1999) 113; J.M. Nester, F. H. Ho and C. M.Chen, Quasilocal Center-ofMass for Teleparallel Gravity, Proceeding of the 10th. Marcel Grossman Meeting (Rio de Janeiro, 2003) gr-qc/0403101; J. M. Nester, Phys. Lett. A139 (1989) 112; J. Math. Phys. 33 (1992), 910; Class. Quantum Grav. 5 (1988), 1003..

[26] N. Toma, Prog. Theor. Phys. 86 (1991), 659; T. Kawai and N. Toma, Prog. Theor. Phys. 87 (1992), 583 .

[27] V.C. de Andrade and J.G. Pereira, Phys. Rev. D56 (1997), 4689; V.C. de Andrade, L.C.T Guillen and J.G. Pereira, Phys. Rev. Lett. 84 (2000), 4533; Phys. Rev. D64 (2001), 027502.

[28] J.W. Maluf and J.F. da Rocha-Neto, Phy. Rev. D64 (2001), 084014.

[29] J. Schwinger, Phys. Rev. 130 (1963), 1253.

[30] J. W. Maluf, J. Math. Phys. 35 (1994), 335; J. W. Maluf and A. Kneip, J. Math. Phys. 38 (1997), 458; J. W. Maluf and J. F. da Rocha-Neto, J. Math. Phys. 40 (1999), 1490; J. W. Maluf and A. Goya, Class. Quant. Grav. 18 (2001), 5143; J. W. Maluf, J. F. da Rocha-Neto, T. M. L. Toribio and K. H. Castello-Branco, Phys. Rev. D65 (2002), 124001; A. A. Sousa and J. W. Maluf, Prog. Theor. Phys. 108 (2002), 457.

[31] J.F. da Rocha-Neto and K. H. Castello-Branco, JHEP 0311 (2003), 002.

[32] T. Regge and C. Teitelboim, Ann. Phys. (New York) 88 (1974), 286.

[33] J. W. York, Jr., Energy and Momentum of the Gravitational field, in "Essays in General Relativity" edited by F.J. Tipler (Acdemic Press, New York, 1980).

[34] R. Beig and N. ó Murchadha, Ann. Phys. (New York) 174 (1987), 463.

[35] L.B. Szabados, Class. Quantum. Grav. 20 (2003) 2627.

[36] J. W. Maluf and A.A. Sousa, Hamiltonian formulation of teleparallel theories of gravity in the time gauge gr-qc/0002060, (2000).

[37] A. A. Sousa and J. W. Maluf, Prog. Theor. Phys. 104 (2000), 531.

[38] J. W. Maluf and J. F. de Rocha-Neto, Phys. Rev. D64 (2001), 084014.

[39] J.W. Maluf, S.C. Ulhoa, F.F. Faria and J.F. da Rocha-Neto, Calss. Quant. Grav. 23 (2006), 6245 .

[40] A. A. Sousa, R.B. Pereira and J.F. da Rocha-Neto, Prog. Theor. Phys. 114 (2005), 1179; A. A. Sousa, J.S. Moura and R.B. Pereira gr-qc/0702109. 
[41] Shanxian Xu and Jiliang Jing, it Class. Quant. Grav. 23 (2006), 4659.

[42] J. M. Nester, Int. J. Mod. Phys. A4 (1989), 1755.

[43] M. Blagojević and M. Vasilić Phys. Rev. D64 (2001), 044010.

[44] C.C. Change, J.M. Nester, Grav. \& Cosmol. 6 (2000) 257.

[45] L.L. So, J.M. Nester and H. Chen, The 7th. conference on Gravitation and Astrophysics; gr-qc/0605150.

[46] L.B. Szabados, "Quasi-Local Energy-Momentum and Angular Momentum in GR: A Review Article", Living Rev. Relativity 7, (2004), 4. http://www.livingreviews.org/lrr2004-4.

[47] J. W. Maluf, Annalen Phys. 14 (2005), 723; Gravitational and Cosmology 11 (2005), 284.

[48] D. Christodoulou, Phys. Rev. Lett. 25 (1970), 1596.

[49] G. Bergqvist, Class. Quant. Grav. 9 (1992), 1753.

[50] A. Komar, Phys. Rev. 113 (1959), 934; J. Winicour, " Angular Momentum in General Relativity", in General Relativity and Gravitation edited by A. Held (Plenum, New York, 1980); A. Ashtekar, " Angular Momentum of Isolated Systems in General Relativity", in Cosmology and Gravitation, edited by P.G. Bergmann and V. de Sabbata (Plenum, New York 1980).

[51] K. Hayashi and T. Shirafuji, Prog. Theor. Phys. 73 (1985), 54; M. Blagojević and M. Vasilić Class. Quant. Grav. 5 (1988), 1241; T. Kawai, Phys. Rev. D62 (2000), 104014, T. Kawai, K. Shibata and I. Tanaka, Prog. Theor. Phys. 104 (2000) 505.

[52] P.A.M. Dirac, Lectures on Quantum Mechanics (Belfer Gradute School of Science (Monographs Series No. 2) Yeshiva University, New York, 1964).

[53] H.P. Robertson, Ann. of Math. (Princeton) 33 (1932), 496.

[54] K. Hayashi and T. Shirafuji, Phys. Rev. D19 (1979), 3524.

[55] T. Shirafuji, G.G.L. Nashed, and K. Hayashi, Prog. Theor. Phys. 95 (1996), 665.

[56] M. Salti and O. Aydoĝdu M. Korunur, will appear in JHEP; gr-qc/0611014.

[57] R Arnowitt, S. Deser and C.W. Misner, Gravitation: An Introduction to Current Research edited by L. Witten (Wiley, N.Y. (1962)).

[58] C.W. Misner, K.S. Thorne and J.A. Wheeler, Gravitation (Freeman, San Francisco, 1973), P. 435.

[59] R. Casadio, A. Fabbri and L. Mazzacurati, Phys. Rev. D65 (2002), 084040.

[60] C. Germani and R. Maartens, Phys. Rev. D64 (2001), 124010.

[61] K.A. Bronnikov and S.-W. Kim, Phys. Rev. D67 (2003), 064027. 
[62] J. W. Maluf, M.V.O. Veiga and J. F. da Rocha-neto, Gen. Rel. Grav. 39 (2007), 227.

[63] P. Bae(c)kler, R. Hecht, F.W. Hehl and T. Shirafuji, Prog. Theor. Phys. 78 (1987), 16.

[64] J.D. Brown and J.W. York, Jr., Quasi-local energy in general relativity, Proceedings of the Joint Summer Research conference on Mathematical Aspects of Classical Field Theory, edited by M.J. Gotay, J.E. Marsden and V. Moncrief (American Mathematical Socity, 1991); Phys. Rev. D47 (1993), 1407.

[65] M. Sharif and M. Jamil Amir, Mod. Phys. Lett. A 23 (2008), 969.

[66] D. Kramer, H. Stephani, M.A.H. MacCullum and E. Herlt, Exact solutions of Einstein field equations, Cambridge Univ. Press, Cambridge, 1980.

[67] G.S. Hall, Symmetryies and curvature structure in general relativity, World Scientific , Singapore, 2004.

[68] T. Feroze, F.M. Mahomed and A. Qadir, Nonlinear Dynam. 45 (2006), 65.

[69] C. Møller, Mat. Fys. Medd. Dan. Vid. Selsk. 35 (1966), no.3. 